\title{
INFLUÊNCIA DO HOSPEDEIRO NO PERFIL POPULACIONAL E NAS POPULAÇÕES DE CILIADOS DO RÚMEN DE BOVINOS
}

\author{
Marta D'Agosto ${ }^{1}$ \\ Milden Rodrigues de Santa-Rosa ${ }^{2}$
}

\begin{abstract}
HOST INFLUENCE ON THE POPULATION PROFILE AND ON THE CILIATE POPULATIONS OF THE BOVINE RUMEN. The influence of the host on the population profile and on the ciliate populations of the bovine rumen was investigated using three suckling crossbred friesian-zebu (Gir) cows, with fistulas. The genera of ciliate observed in the three hosts examined have characterized the populations as type A. The occurrence of the genera of ciliate was related to the host. It was verified that the rumen ciliate were quantitatively influenced by inherent factors in the host animals, showing a significant difference in the total of ciliate among the hosts in all stages of the experiment.

KEY WORDS. Entodiniomorphida, Isotrichidae, Blepharocorythidae, bovine, rumen ciliate populations
\end{abstract}

Estudos sobre competição e antagonismo entre ciliados do rúmen realizados por EADIE (1962a, 1962b) demonstraram que Polyplastron multivesiculatum (Dogiel \& Fedorowa, 1925), Eudiplodinium maggii (Fiorentini, 1889) e Epidinium spp., não estabelecem populações mistas. Com base nestas observações, foram propostas as seguintes designações: a) população do tipo A, com P. multivesiculatum dentre outros ciliados, tais como Diploplastron affine (Dogiel \& Fedorowa, 1925); b) população do tipo B, na qual E. maggii e Epidinium spp., juntos ou isolados, são os grandes entodiniomorfos predominantes. Esta caracterização de populações baseiase no antagonismo entre algumas espécies de ciliados já relatadas por outros autores (EADIE 1967; ABOU AKKADA et al. 1969; JOUANY et al. 1988). Há ainda a população do tipo 0, conforme assinalada por TownE et al. (1988), com a presença apenas de Entodinium spp. e/ou isotriquídeos.

Além do antagonismo, fatores metabólicos individuais dos hospedeiros podem exercer influencia na ocorrência e na concentração dos ciliados do rúmen (PURSER \& Morr 1966; Towne et al. 1988; Williams \& Coleman 1991).

O presente trabalho objetivou realizar um levantamento do perfil populacional de ciliados do rúmen em bovinos e avaliar se esses ciliados foram qualitativa e quantitativamente influênciados por fatores inerentes aos animais hospedeiros.

1) Departamento de Zoologia, Instituto de Ciências Biológicas, Universidade Federal de Juiz de Fora. 36036-330 Juiz de Fora, Minas Gerais, Brasil.

E-mail: dagosto@icb.ufjf.br

2) Instituto de Microbiologia, Universidade Federal do Rio de Janeiro. 21944-970 Rio de Janeiro, Rio de Janeiro, Brasil. 


\section{MATERIAL E MÉTODOS}

O trabalho foi desenvolvido no Centro Nacional de Pesquisa de Gado de Leite (CNPGL)/Embrapa, situado no Município de Coronel Pacheco, Minas Gerais e no Departamento de Zoologia da Universidade Federal de Juiz de Fora.

\section{Animais}

Foram utilizadas três vacas mestiças Gir-holanda com mesmo grau sangüíneo (A1, A2 e A3), em lactação, fistuladas, com idade de seis anos e peso entre 470 e $500 \mathrm{~kg}$, mantidas no CNPGL.

\section{Dietas}

Antes de se iniciar o trabalho, os animais foram alimentados com silagem de milho, capim e concentrado proteico, fornecido duas vezes ao dia. Em preparação para o experimento, receberam durante uma semana, pela manhã, $36 \mathrm{Kg}$ de silagem de milho mais $2 \mathrm{Kg}$ de farelo de algodão (Dieta $0=\mathrm{D} 0$ ). Água foi fornecida $a d$ libitum.

As dietas (D1, D2, D3), constituídas à base de ração (57\% de fubá de milho, $40 \%$ de farelo de soja, $02 \%$ de calcáreo e $01 \%$ de sais minerais) e silagem de milho, tiveram a seguinte relação entre a massa seca $(\mathrm{ms})$ da silagem e da ração: (D1) = $30,0 \mathrm{~kg}$ de silagem de milho $(80 \% \mathrm{~ms})+2,5 \mathrm{~kg}$ de ração $(20 \% \mathrm{~ms}) ;(\mathrm{D} 2)=25,25$ $\mathrm{kg}$ de silagem de milho $(70 \% \mathrm{~ms})+3,75 \mathrm{~kg}$ de ração $(30 \% \mathrm{~ms}) ;(\mathrm{D} 3)=22,5 \mathrm{~kg}$ de silagem de milho $(60 \% \mathrm{~ms})+5,0 \mathrm{~kg}$ de ração $(40 \% \mathrm{~ms})$.

\section{Experimento}

O experimento constou de três fases (F1, F2, F3), com duração de uma semana cada, precedidas por períodos de adaptação de duas semanas, nos quais as dietas a serem testadas (D1, D2, D3) foram introduzidas. Em F1 cada animal recebeu uma das três dietas, sendo que nas fases subseqüentes as dietas foram permutadas entre os animais.

O delineamento experimental utilizado foi o quadrado latino, considerando três tratamentos (variações na dieta) e três animais com repetições no tempo (fases).

Para a análise estatística utilizou-se o SAS (Statistical Analysis Sistem), calculando-se médias com desvios padrão. Foram feitas análises de variância seguidas do teste de Tukey a 5\%.

\section{Obtenção e exame das amostras}

Foram obtidas 270 amostras de conteúdo ruminal através de fístulas, após 15 dias da introdução da dieta. Todas as amostras foram recolhidas pela manhã, antes de serem fornecidas as dietas. Em cada fase do experimento foram obtidas, em duas coletas, 30 amostras de conteúdo ruminal por hospedeiro. Outras três amostras de conteúdo ruminal, uma de cada animal, foram recolhidas enquanto estes recebiam D0, como base de comparação do efeito da dieta sobre os ciliados.

As amostras consistiram de $20 \mathrm{ml}$ de líquido ruminal, obtido por aspiração com bomba de bicicleta com a válvula invertida, acrescido de $20 \mathrm{~cm}^{3}$ de conteúdo ruminal, recolhido do centro da massa ruminal. Estas amostras foram mantidas em recipientes individualizados, homogenizadas, fixadas na proporção 1: 1 em forma- 
lina a 18,5\% (DEHORITY 1984) e conservadas em frascos vedados devidamente identificados. Logo após a obtenção das amostras, verificou-se o pH de cinco amostras de cada animal, escolhidas aleatoriamente.

Avaliações qualitativas e quantitativas dos ciliados ruminais foram realizadas no laboratório do Departamento de Zoologia da Universidade Federal de Juiz de Fora, segundo técnica proposta por DEHORITY (1984) modificada por D'AGOSTO \& CARNEIRO (1995). As contagens foram precedidas por observações dos ciliados por meio de preparações provisórias em lâmina e lamínula, coradas com solução de lugol e com verde de metila, para possibilitar identificação mais segura na câmara de contagem.

Os resultados das contagens expressam o número de ciliados por mililitro de conteúdo ruminal.

A identificação dos ciliados baseou-se em OGimoto \& IMAI (1981). Para a identificação dos gêneros de Diplodiinae adotou-se a proposta de KOFOID \& MACLENNAN (1932), também seguida por OGIMOTO \& IMAI (1981).

\section{RESULTADOS}

No conteúdo ruminal dos três bovinos foram detectados e quantificados ciliados das famílias Blepharocorythidae (Charonina Strand, 1928), Isotrichidae (Isotricha Stein, 1859, Dasytricha Schuberg, 1888) e Ophryoscolecidae (Entodiinae: Entodinium Stein, 1859; Diplodiinae: Diplodinium Schuberg, 1888, Eodinium Kofoid \& MacLennan, 1932, Eremoplastron Kofoid \& MacLennan, 1932, Polyplastron Dogiel, 1927, Diploplastron Kofoid \& MacLennan, 1932 e Ostracodinium Dogiel, 1927, sendo os três últimos gêneros analisados em conjunto e referidos como $P+D+O)$.

À exceção de Dasytricha, só registrado em A1, e de Eremoplastron, presente em A1 e em A2, esses ciliados foram observados nos três animais em todas as fases do experimento. A presença dos gêneros foi constante para os três animais em todas as dietas e/ou fases, mesmo enquanto os animais recebiam D0, excetuando-se Isotricha e Dasytricha que não foram registrados em A1 em D0.

$\mathrm{O}$ número médio de ciliados observados por famílias e por subfamílias de Ophryoscolecidae em A1, A2 e A3 está apresentado na tabela I. Nota-se que a família Ophryoscolecidae prevaleceu sobre Blepharocorythidae e Isotrichidae, representando $93,14 \%, 96,67 \%$ e $97,67 \%$ em A1, A2 e A3, respectivamente, do total de ciliados observados em cada animal. Destaca-se ainda, que os organismos da subfamília Entodiinae representaram $83,52 \%$ do total de Ophryoscolecidae.

$\mathrm{Na}$ tabela II estão apresentados os grupos de ciliados e respectivos valores médios e totais, valores mínimo e máximo, desvio padrões e percentuais em relação ao total de protistas por animal.

$\mathrm{O}$ número médio total de protistas por hospedeiro revelou maior concentração de ciliados em A2 com 46,11\% do total, seguido por A 1 (30,29\%) e A3 (23,61\%) (Tab. III). A tabela III apresenta ainda os dados de cada gênero de ciliado e respectivos percentuais em relação ao total por hospedeiro, destacando-se a predominância de Entodinium sobre os outros ciliados, representando 83,22\%, 78,04\% e $94,99 \%$ do total em A1, A2 e A3, respectivamente. 
Tabela I. Valores médios e totais de ciliados do rúmen de três bovinos (por mililitro de conteúdo ruminal) e respectivos percentuais, por familias dos organismos observados e por subfamílias de Ophryoscolecidae.

\begin{tabular}{|c|c|c|c|c|c|c|}
\hline \multicolumn{3}{|c|}{ Ciliados } & \multicolumn{3}{|c|}{ Bovino } & \multirow{2}{*}{ Totais } \\
\hline Familias & & Subfamilias & $\mathrm{A} 1^{\star \star *}$ & $A 2^{* *}$ & $\mathrm{~A} 3^{\star \star}$ & \\
\hline \multirow[t]{2}{*}{ Blepharocorythidae } & $\left(n^{\circ}\right)$ & & $12.364,44$ & $8.404,44$ & $2,751,11$ & $23.520,0 \mathrm{C}$ \\
\hline & $(\%)$ & & 4,82 & 2,19 & 1,93 & 2,78 \\
\hline \multirow[t]{2}{*}{ Isotrichidae } & $\left(n^{\circ}\right)$ & & $5.204,45$ & $7.124,44$ & $1.906,67$ & $14.235,66$ \\
\hline & $(\%)$ & & 2,03 & 1,82 & 0,96 & 1,68 \\
\hline \multirow{6}{*}{ Ophryoscolecidae } & $\left(n^{\circ}\right)$ & & $238.706,67$ & $374.586,66$ & $192.075,55$ & $808,368,88$ \\
\hline & $(\%)$ & & 93,14 & 96,02 & 97,67 & 95,54 \\
\hline & & Entodiinae $\left(n^{\circ}\right)$ & $213.280,00$ & $304.431,11$ & $188.982,22$ & $706.693,33$ \\
\hline & & $(\%)$ & 89,35 & 81,27 & 96,88 & 87,42 \\
\hline & & Diplodiinae $\left(n^{\circ}\right)$ & $25.426,67$ & $70.155,55$ & $6.093,33$ & $101.675,55$ \\
\hline & & $(\%)$ & 10,65 & 18,73 & 3,12 & 12,58 \\
\hline Totais * & & & $256.275,56$ & $390.115,56$ & $199.733,33$ & $846,124,44$ \\
\hline
\end{tabular}

*) Referem-se às somas dos ciliados registrados nas familias; ${ }^{\star \star}$ ) vacas Gir-holanda com mesmo grau sangüíneo.

Tabela II. Valores mínimos, máximos, médios, percentuais e desvios padrão de cada gênero de ciliados do rúmen de três bovinos (por mililitro de conteúdo ruminal), por hospedeiro.

\begin{tabular}{|c|c|c|c|c|c|c|c|c|c|c|c|c|}
\hline \multirow{2}{*}{ Ciliados } & \multicolumn{4}{|c|}{$\mathrm{A} 1^{* *}$} & \multicolumn{4}{|c|}{$\mathrm{A} 2^{* *}$} & \multicolumn{4}{|c|}{$\mathrm{A} 3^{* *}$} \\
\hline & Minimo & Máximo & Média & $\begin{array}{l}\text { Desvio } \\
\text { padrão }\end{array}$ & Minimo & Máximo & Média & $\begin{array}{l}\text { Desvio } \\
\text { padrăo }\end{array}$ & Minimo & Máximo & Média & $\begin{array}{l}\text { Desvio } \\
\text { padrăo }\end{array}$ \\
\hline Entodinium & 83200 & 368000 & $\begin{array}{r}213280,54 \\
83,22\end{array}$ & 63199,54 & 161600 & 497600 & $\begin{array}{r}304431,11 \\
78,04\end{array}$ & 65283,23 & 56000 & 416000 & $\begin{array}{r}188982,22 \\
94,62\end{array}$ & 84329,78 \\
\hline Isotrhicha & 0 & 17600 & $\begin{array}{r}4075,56 \\
1,59\end{array}$ & 4184,23 & 0 & 20800 & $\begin{array}{r}7124,44 \\
1,83\end{array}$ & 3942,04 & 0 & 11200 & $\begin{array}{r}1906,67 \\
0,96\end{array}$ & 1976,40 \\
\hline Dasytricha & 0 & 8000 & $\begin{array}{r}1128,89 \\
0,44\end{array}$ & 1669,34 & 0 & 0 & $\begin{array}{l}0,00 \\
0,00\end{array}$ & 0,00 & 0 & 0 & $\begin{array}{l}0,00 \\
0,00\end{array}$ & 0,00 \\
\hline Diplodinium & 0 & 25600 & $\begin{array}{r}6813,33 \\
2,66\end{array}$ & 4650,58 & 1600 & 81600 & $\begin{array}{r}26973,33 \\
6,91\end{array}$ & 13366.24 & 0 & 8000 & $\begin{array}{r}1324,44 \\
0,66\end{array}$ & 1708,46 \\
\hline$P .+D .+O{ }^{*}$ & 0 & 27200 & $\begin{array}{r}8728,89 \\
3,41\end{array}$ & 4426,14 & 0 & 44800 & $\begin{array}{r}12400,00 \\
3,20\end{array}$ & 6168,28 & 0 & 12800 & $\begin{array}{r}2506,67 \\
1,26\end{array}$ & 2093,14 \\
\hline Eremoplastron & 0 & 16000 & $\begin{array}{r}2915,56 \\
1,14\end{array}$ & 2889,22 & 4800 & 73600 & $\begin{array}{r}24542,22 \\
6,29\end{array}$ & 12354,55 & 0 & 0 & $\begin{array}{l}0,00 \\
0,00\end{array}$ & 0,00 \\
\hline Eodinium & 0 & 30400 & $\begin{array}{r}6968,89 \\
2,72\end{array}$ & 4912,69 & 0 & 20800 & $\begin{array}{r}6160,00 \\
1,58\end{array}$ & 3823,31 & 0 & 16000 & $\begin{array}{r}2262,22 \\
1,13\end{array}$ & 2267,98 \\
\hline Charonina & 0 & 46400 & $\begin{array}{r}12364,44 \\
4,82\end{array}$ & 8562,43 & 0 & 32000 & $\begin{array}{r}8404,44 \\
2,15\end{array}$ & 5459,60 & 0 & 14400 & $\begin{array}{r}2751,11 \\
1,38\end{array}$ & 3327,45 \\
\hline Totais & & & 256275,56 & & & & 390115,56 & & & & 199733,33 & \\
\hline
\end{tabular}

*) Polyplastron + Diploplastron + Ostracodinium; $n=90 ;{ }^{\star \star}$ ) vacas Gir-holanda com mesmo grau sangüíneo.

Em A1, Charonina foi o segundo gênero mais observado (4,82\%) enquanto Dasytricha foi o de menor concentração $(0,44 \%)$ e só esteve presente neste animal. Já em A2, destacaram-se os organismos dos gêneros Diplodinium $(6,91 \%)$ e Eremoplastron (6,29\%) sendo este último valor expressivo para A2, por representar $89,38 \%$ do registro destes organismos nos animais em que esteve presente.

Ao se observar a posição relativa de cada gênero de ciliados em cada hospedeiro com relação ao registro total destes gêneros, destacaram-se em A2, além da elevada representação absoluta e percentual de Eremoplastron, as maiores concentrações de Entodinium, Isotricha, Diplodinium e $P+D+O$. Já em Al observaram-se as mais elevadas concentrações de Dasytricha, Eodinium e Charonina. 
A3 apresentou a menor diversidade de ciliados e os menores valores por gêneros e total em relação aos outros hospedeiros.

As médias das contagens de cada gênero de ciliados variou significativamente entre os três animais em todos os gêneros, à exceção de Eodinium que não diferiu significativamente entre A1 e A2; do mesmo modo as médias das contagens totais dos ciliados variaram significativamente entre A1, A2 e A3 (Tab. III).

Tabela III. Valores médios e totais de ciliados do rúmen de três bovinos (por mililitro de conteúdo ruminal) e respectivos percentuais em relação ao total de ciliados por animal.

\begin{tabular}{|c|c|c|c|c|c|}
\hline & \multirow{2}{*}{ iliados } & \multicolumn{3}{|c|}{ Bovino } & \multirow{2}{*}{ Totais } \\
\hline & & $\mathrm{A} 1^{\star \star *}$ & $\mathrm{~A} 2^{\star \star}$ & $\mathrm{A} 3^{\star *}$ & \\
\hline Entodinium & $\begin{array}{l}\left(n^{\circ}\right) \\
(\%)\end{array}$ & $\begin{array}{c}213.280,00 \mathrm{a} \\
83,22\end{array}$ & $\begin{array}{c}304,431,11 b \\
78,04\end{array}$ & $\begin{array}{c}188.982,22 \mathrm{c} \\
94,62\end{array}$ & $\begin{array}{c}706.693,33 \\
83,52\end{array}$ \\
\hline Isotricha & $\begin{array}{l}\left(n^{\circ}\right) \\
(\%)\end{array}$ & $\begin{array}{c}4.075,56 a \\
1,59\end{array}$ & $\begin{array}{c}7.124,44 \mathrm{~b} \\
1,83\end{array}$ & $\begin{array}{c}1,906,67 \mathrm{c} \\
0,96\end{array}$ & $\begin{array}{c}13.106,67 \\
1,55\end{array}$ \\
\hline Dasytricha & $\begin{array}{l}\left(n^{\circ}\right) \\
(\%)\end{array}$ & $\begin{array}{c}1.128,89 \mathrm{a} \\
0,44\end{array}$ & - & - & $\begin{array}{c}1.128,89 \\
0,13\end{array}$ \\
\hline Diplodinium & $\begin{array}{l}\left(\mathrm{n}^{\circ}\right) \\
(\%)\end{array}$ & $\begin{array}{c}6.813,33 a \\
2,66\end{array}$ & $\begin{array}{c}26.973,33 b \\
6,91\end{array}$ & $\begin{array}{c}1.324,44 \mathrm{c} \\
0,66\end{array}$ & $\begin{array}{c}35.111,10 \\
4,15\end{array}$ \\
\hline$P+D+0$. & $\begin{array}{l}\left(n^{\circ}\right) \\
(\%)\end{array}$ & $\begin{array}{c}8.728,89 \text { a } \\
3,41\end{array}$ & $\begin{array}{c}12.480,00 b \\
3,20\end{array}$ & $\begin{array}{c}2.506,67 \mathrm{c} \\
1,26\end{array}$ & $\begin{array}{c}23.715,56 \\
.2,80\end{array}$ \\
\hline Eremoplastron & $\begin{array}{l}\left(\mathrm{n}^{\circ}\right) \\
(\%)\end{array}$ & $\begin{array}{c}2.915,56 \mathrm{a} \\
1,14\end{array}$ & $\begin{array}{c}24,542,22 b \\
6,29\end{array}$ & - & $\begin{array}{c}27.457,78 \\
3,25\end{array}$ \\
\hline Eodinium & $\begin{array}{l}\left(\mathrm{n}^{\circ}\right) \\
(\%)\end{array}$ & $\begin{array}{c}6.968,89 a \\
2,72\end{array}$ & $\begin{array}{c}6.160,00 \mathrm{a} \\
1,58\end{array}$ & $\begin{array}{c}2.262,22 b \\
1,13\end{array}$ & $\begin{array}{c}15.391,11 \\
1,82\end{array}$ \\
\hline Charonina & $\begin{array}{l}\left(n^{\circ}\right) \\
(\%)\end{array}$ & $\begin{array}{c}12,364,44 \mathrm{a} \\
4,82\end{array}$ & $\begin{array}{c}8.404,44 b \\
2,15\end{array}$ & $\begin{array}{c}2.751,11 \mathrm{c} \\
1,38\end{array}$ & $\begin{array}{c}23.519,99 \\
2,78\end{array}$ \\
\hline Totais & & $\begin{array}{c}256.275,56 \text { a } \\
30,29\end{array}$ & $\begin{array}{c}390,115,56 b \\
46,11\end{array}$ & $\begin{array}{c}199.733,33 \mathrm{c} \\
46,11\end{array}$ & $846,124,43$ \\
\hline
\end{tabular}

*) Polyplastron + Diploplastron + Ostracodinium; médias nas linhas, seguidas por letras diferentes, diferem a um nivel de significância de $5 \%$; ${ }^{* *}$ ) vacas Gir-holanda com mesmo grau sangüíneo.

Na tabela IV estão apresentados os dados referentes aos totais de ciliados registrados e em cada dieta, por animal, com os respectivos percentuais em relação a estes totais, observando-se a maior concentração de ciliados em A2, seguido de A1. Ao se analisar os dados dos animais em relação a cada uma das dietas notou-se que esta correspondência foi mantida, com A2 apresentando as maiores concentrações de ciliados em todas as dietas, seguido por A1, exceto em D1, quando A3 apresentou maior número de ciliados que $\mathrm{A} 1$. As proporções mais elevadas foram observadas em D2 para A1 e A3 e em D3 para A2. Nesta tabela, estão apresentados, ainda, os valores médios do $\mathrm{pH}$ de cinco amostras de cada coleta, por animal.

Ao se analisar as relações percentuais dos ciliados observados em Al, A2 e A3 com dietas iguais (Tab. IV), confirmou-se a predominância de ciliados em A2 em todas as dietas.

\section{DISCUSSÃO}

Os gêneros de ciliados do rúmen registrados em Al, e o registro semelhante nos outros animais, excetuando-se organismos do gênero Dasytricha em A2 e de Dasytricha e Eremoplastron em A3, permitiu o reconhecimento da ocorrência de 
população do tipo A nos três hospedeiros. Segundo EADIE (1962b), além de espécies de Entodinium e de isotriquídeos, comuns aos dois tipos de populações assinaladas por este autor, a população do tipo A caracteriza-se pela ocorrência de Polyplastron multivesiculatum, geralmente associada a Diploplastron affine, como registrado no presente trabalho, ou a Ophryoscolex tricoronatus. Apesar de ter sido caracterizado como do tipo A, o conjunto de ciliados detectados apresentou organismos que geralmente estão associados a outro tipo de população. Desta forma, registrou-se a ocorrência de Ostracodinium nos três animais e de Eremoplastron em A1 e A2. Estes organismos geralmente coexistem com Eudiplodinium maggi e Epidinium spp., constituindo a chamada população do tipo B. Todavia, o registro de população estável de Eremoplastron junto com Polyplastron já havia sido relatado por EADIE (1962b), e o de Ostracodinium em populações mistas A-B com a presença de Polyplastron, em bisões, por Towne et al. (1988) e a coexistência destes últimos em ovinos por DEHORITY (1978), o que respalda a presente caracterização como população do tipo A.

Tabela IV. Valores médios e totais de ciliados do rúmen de bovinos (por $\mathrm{ml}$ de conteúdo ruminal), de acordo com a dieta e o hospedeiro, e respectivos percentuais em relação ao total de ciliados por animal, e pH médio de cada situação.

\begin{tabular}{|c|c|c|c|c|c|c|c|c|}
\hline & \multirow{2}{*}{ Dieta * } & \multicolumn{6}{|c|}{ Bovino } & \multirow{2}{*}{ Totais } \\
\hline & & $\mathrm{A} 1^{*+}$ & $\mathrm{pH}$ & $\mathrm{A} 2^{* *}$ & $\mathrm{pH}$ & $\mathrm{A} 3^{* *}$ & $\mathrm{pH}$ & \\
\hline \multirow[t]{2}{*}{ D1 } & $\left(n^{\circ}\right)$ & $186.226,67$ & 6,98 & $340.760,00$ & 6,98 & $231.013,33$ & 6,40 & $758.000,00$ \\
\hline & $(\%)$ & 24,22 & $+0,13$ & 29,12 & $+0,24$ & 38,55 & $+0,20$ & 29.86 \\
\hline \multirow[t]{2}{*}{ D2 } & $\left(n^{\circ}\right)$ & $331.173,33$ & 7,35 & $370.000,00$ & 6,97 & $247.626,67$ & 5,76 & $948.800,00$ \\
\hline & $(\%)$ & 43,08 & $+0,07$ & 31,62 & $+0,16$ & 41,33 & $+0,66$ & 37,38 \\
\hline \multirow[t]{2}{*}{ D3 } & $\left(n^{\circ}\right)$ & $251,426,67$ & 6,83 & $459.586,67$ & 7,30 & $120.560,00$ & 6,51 & $831.573,34$ \\
\hline & $(\%)$ & 32,70 & $+0,33$ & 39,27 & $+0,31$ & 20,12 & $+0,16$ & 32,34 \\
\hline \multirow[t]{2}{*}{ Totais } & $\left(n^{\circ}\right)$ & $768.826,67$ & & $1.170 .346,67$ & & $599.200,00$ & & $2.538 .373,34$ \\
\hline & $(\%)$ & 30,29 & & 46,11 & & 23,61 & & \\
\hline
\end{tabular}

*) (D1) $30,0 \mathrm{~kg}$ de silagem de milho + 2,5 kg de ração; (D2) 25,5 kg de silagem de milho + $3,75 \mathrm{~kg}$ de ração; (D3) $22,5 \mathrm{~kg}$ de silagem de milho $+5,0 \mathrm{~kg}$ de ração; **) vacas Gir-holanda com mesmo grau sangüíneo.

A distribuição dos gêneros e respectivas concentrações observadas, possivelmente relacionam-se aos valores do $\mathrm{pH}$, tendo sido os mais baixos registrados em A3, que foi o hospedeiro que apresentou as menores concentrações e a menor diversidade de ciliados. Estas considerações concordam com o observado por PURSER \& MOIR (1959) quanto à inibição da reprodução de ciliados do rúmen nos períodos diurnos de $\mathrm{pH}$ mais baixo. Tanto a instalação quanto a manutenção das populações de ciliados do rúmen parecem estar relacionadas ao $\mathrm{pH}$, conforme já relatado por PURSER \& MOIR (1966), EAdIE (1962a), NogUEIRA FILHO et al. (1984) e Oliverra et al. $(1987,1989)$. Provavelmente a instalação precoce de Entodinium, assinalada em bezerros com dois meses de idade e pH de 4,2 por NOGUEIRA FILHO et al. (1984), deva-se, dentre outros fatores, à sua tolerância a esta variação. Outros autores assinalaram que com pH ruminal abaixo de 6,0 (DEHORITY 1987) as espécies de Entodinium constituiam basicamente o total de ciliados presentes. $\mathrm{O}$ baixo $\mathrm{pH}$, possivelmente associado a outros fatores individuais não investigados, podem ter 
determinado as altas concentrações de Entodinium, sobretudo em A3, com 94,62\% do total de protistas detectados neste animal (Tab. III).

No presente trabalho foram assinaladas diferenças significativas no total de ciliados e em cada gênero analisado entre os três animais examinados, demonstrando que há influência individual na ocorrência e no número de ciliados. Isto é demonstrado pela ocorrência de cada gênero de ciliado ter se mantido constante nos três animais, nas diversas situações. Do mesmo modo, a concentração total de ciliados foi maior em A2, independente das dietas e fases, e os menores valores, com única exceção, foram os apresentados por A3 (Tabs I-IV). Estas observações concordam com as de TownE et al. (1988), que assinalaram grande variação na concentração de ciliados entre hospedeiros mantidos com a mesma dieta.

No Brasil, em levantamento de ciliados do rúmen em quatro vacas Nelore, DEHORITY (1986) assinalou variações no total de ciliados, relacionadas ao hospedeiro, de $9,0 \times 10^{4}$ a $51,2 \times 10^{4}$. As variações registradas por este autor refletiram-se também em cada família de ciliados observadas, até mesmo na subfamília Entodiinae, cujos percentuais variaram entre $42,2 \%$ e $84,6 \%$. Nos registros ora apresentados, apesar das contagens totais diferenciadas, os percentuais destes organismos foram elevados em todos os hospedeiros, representando $78,04 \%$ a $94,62 \%$ do total de ciliados por animal (Tab. I).

Todas as variações assinaladas no presente trabalho, tanto no perfil populacional quanto na concentração de ciliados do rúmen por hospedeiro, indicam que além da influência das dietas, fatores inerentes ao metabolismo dos hospedeiros não podem ser desprezados. Estes resultados respaldam as considerações de WiLLiaMS \& COLEMAN (1991) que destacaram a importância da influência individual do hospedeiro ao afirmar que resultados, nos quais efeito de diferentes regimes alimentares foram determinados usando-se poucos animais, devem ser tratados com cuidado.

AGRADECIMENTOS. Os autores agradecem ao Centro Nacional de Pesquisa de Gado de Leite (CNPGL/EMBRAPA), especialmente aos pesquisadores Dr. Luís Januário Aroeira, pela cessão dos animais e prescrição das dietas, e Dr. Marcus Cordeiro Durães, pela análise estatística.

\section{REFERÊNCIAS BIBLIOGRÁFICAS}

Abou AKKadA, A.R.; E.E. Bartley \& L.R. Fina. 1969. Ciliate protozoa in the rumen of the lactating cow. Jour. Dairy Sci. 52: 1088-1091.

D'AGosto, M. \& M.E. CARNEIRO. 1995. Avaliação do emprego da solução de lugol na quantificação de ciliados do rúmen. Rev. Bras. Parasitol. Vet. 4: 171.

DEHORITY, B.A. 1978. Specificity of rumen ciliate Protozoa in cattle and sheep. Jour. Protozool. 25: 509-513.

1984. Evaluation of subsampling and fixation procedures used for counting rumen Protozoa. Appl. Environ. Microbiol. 48: 182-185.

. 1986. Rumen ciliate fauna of some brazilian cattle: occurence of several ciliates new to the rumen, including the cycloposthid Parentodinium africanum. 
Jour. Protozool. 33: 416-421.

1987. Rumen Ophryoscolecid Protozoa in the hindigut of the Capaybara

(Hydrochoerus hydrochaeris). Jour. Protozool. 34 (2): 143-145.

EADIE, M. 1962a. The development of rumen microbial populations in lambs and calves under various conditions of management. Jour. Gen. Microbiol. 29: 563-578.

1962b. Inter-relationships between certain rumen ciliate protozoa. Jour.

Gen. Microbiol. 29: 579-588.

1967. Studies on the ecology of certain rumen ciliate protozoa. Jour. Gen.

Microbiol. 49: 175-194.

JoUANY, J.P.; D.I. DEMEYER \& J. GRAIN. 1988. Effect of defaunating the rumen. Anim. Feed Sci. Technol. 21: 229-265.

Kofoid, C.A. \& R.F. MacLennan. 1932. Ciliates from Bos indicus Linn. II. A revision of Diplodinium Schuberg. Univ. Calif. Publ. Zool. 37: 53-152.

Nogueira Filho, J.C.M.; M.E.M. Oliveira; J.S.M. VeIgA \& C.S. LuCCI. 1984. Cronologia do aparecimento de protozoários ciliados no rúmen de bezerros do tipo "Mantiqueira" (Bos taurus L.), na região do Vale do Rio Paraíba, SP. Rev.

Fac. Med. Vet. Zootec. Univ. S. Paulo. 21: 119-123.

Ogimoto, K.S. Imai. 1981. Atlas of Rumen Microbiology. Tokyo, Japan Scientific Societies Press, VIII+231p.

Oliveira, M.E.M. DE; J.C.M. Nogueira FilHo; C. DE S. LuCCI \& C.G. DE LiMA. 1987. Desenvolvimento de população de protozoários ciliados no rúmen de ovinos (Ovis aries L.) criados em Itapetininga, São Paulo. Rev. Fac. Med. Vet. Zootec. Univ. São Paulo. 24: 225-232.

OLIVEIRA, M.E.M. DE; J.C.M. NOGUEIRA FILHO \& C.S. LUCCI. 1989. Observações sobre o comportamento de populações de protozoários ciliados do rúmen de caprinos (Capra hircus L.) criados em Itapetininga, São Paulo. Rev. Fac. Med. Vet. Zootec. Univ. São Paulo. 26: 15-20.

PURSER, D.B. \& R.J. MoIR. 1959. Ruminal flora studies in sheep. IX. The effect of $\mathrm{pH}$ on the ciliate population of the rumen in vivo. Aust. Jour. Agric. Res. 10: 555-564.

PURSER, D.B. \& R.J. MoIR. 1966. Variations in rumen volume and associated effects as factors influencing metabolism and protozoa concentration in the rumen of sheep. Jour. Anim. Sci. 25: 516-520.

Towne, G.; T.G. NAGARAJA \& K.E. KemP. 1988. Ruminal ciliate in bison. Appl. Environ. Microbiol. 54: 2733-2736.

Williams, A.G. \& G.S. Coleman. 1991. The Rumen Protozoa. Springer-Verlag, New York Inc., 423p.

Recebido em 30.I.1997; aceito em 19.V.1998. 